\title{
Qualidade de vida dos idosos em um município do sertão central cearense
}

\author{
Quality of life of the elderly in a municipality in the central hinterland of Ceará \\ Calidad de vida de los ancianos en un municipio del interior central de Ceará
}

Diego Rodrigues Ponciano ${ }^{1 *}$, Thaís Teles Veras Nunes ${ }^{2}$, Tiago Ribeiro dos Santos ${ }^{3}$, Antonio Germane Alves Pinto ${ }^{3}$, José Adelmo da Silva Filho ${ }^{3}$, Talita Sabrine de Souza ${ }^{3}$, Francisco Arlysson da Silva Verissimo ${ }^{1}$, José de Siqueira Amorim Júnior ${ }^{4}$, Marcos Felipe Genuca da Silva ${ }^{5}$, Vega Vitória Maciel Lopes ${ }^{6}$, Italo Joziel de Morais Guimarães ${ }^{7}$.

\section{RESUMO}

Objetivo: avaliar a qualidade de vida dos idosos de um município do Sertão Central Cearense. Métodos: Trata-se de um estudo epidemiológico do tipo transversal de abordagem quantitativa. A pesquisa foi realizada em um município presente no estado do Ceará, Brasil. A coleta dos dados foi realizada nos meses de março e abril de 2012 com uma amostra de 370 idosos de ambos os sexos. Os dados foram coletados através da aplicação de questionários, evidenciado pela coleta do perfil demográfico, socioeconômico e do perfil clínico. Os dados foram primeiramente agrupados em estratos no Microsoft Office Excel 2007 e tratados estatisticamente utilizando o programa SPSS. Resultados: Os resultados apontam uma diferença significativa entre os sexos, onde os níveis de qualidade para homens são de 53,35\% e para mulheres de 49,73\%. Conclusão: As respostas dessa pesquisa trazem discussões importantes sobre domínios que influenciam na qualidade de vida de idosos e indicam a deficiência de políticas e estratégias de ação que assegurem melhorias de cuidado aos idosos em todas as suas especificidades e diversidade.

Palavras-chave: Idoso, Qualidade de vida, Saúde.

\begin{abstract}
Objective: to evaluate the quality of life of the elderly in a municipality in the Sertão Central Cearense. Methods: This is a cross-sectional epidemiological study with a quantitative approach. The research was carried out in a municipality in the state of Ceará, Brazil. Data collection was carried out in March and April 2012 with a sample of 370 elderly men and women. Data were collected through the application of questionnaires, evidenced by the collection of demographics, socioeconomic and clinical profiles. The data were first grouped into strata in Microsoft Office Excel 2007 and treated statistically using the program SPSS. Results: The results show a significant difference between the sexes, where the levels of quality for men are $53.35 \%$ and for women $49.73 \%$. Conclusion: The responses of this research bring important discussions on domains that influence the quality of life of the elderly and indicate the deficiency of policies and action strategies that ensure improvements in care for the elderly in all their specificities and diversity.
\end{abstract}

Keywords: Elderly, Quality of life, Cheers.

\footnotetext{
1Universidade Estadual do Ceará (UECE), Fortaleza - CE. *E-mail: ponciano.diego1989@gmail.com

${ }^{2}$ Centro Universitário Fametro (Unifametro), Fortaleza - CE.

${ }^{3}$ Universidade Regional do Cariri (URCA), Iguatu - CE.

${ }^{4}$ Universidade Federal do Piauí (UFPI), Picos - PI.

${ }^{5}$ Escola de Saúde Pública do Ceará (ESP), Fortaleza - CE.

${ }^{6}$ Centro Universitário Fanor (UNIFANOR), Fortaleza - CE.

${ }^{7}$ Centro Universitário Ateneu (UNIATENEU), Fortaleza - CE.
} 


\section{RESUMEN}

Objetivo: Evaluar la calidad de vida de los ancianos em um município del Sertão Central Cearense. Métodos: Este es um estudio epidemiológico transversal com un enfoque cuantitativo. La investigación se realizó em un municipio del estado de Ceará, Brasil. La recolección de datos se realizó en marzo y abril de 2012 con una muestra de 370 hombres y mujeres de edad avanzada. Los datos se recopilaron mediante la aplicación de cuestionarios, evidenciados por la recopilación del perfil demográfico, socioeconómico y clínico. Los datos se agruparon primero en estratos en Microsoft Office Excel 2007 y se trataron estadísticamente utilizando el programa SPSS. Resultados: Los resultados muestran una diferencia significativa entre los sexos, donde los niveles de calidad para los hombres son del $53,35 \%$ y para las mujeres del $49,73 \%$. Conclusión: Las respuestas de esta investigación traen debates importantes sobre dominios que influyen en la calidad de vida de los ancianos e indican la deficiencia de políticas y estrategias de acción que garanticen mejoras en la atención a los ancianos en todas sus especificidades y diversidad.

Palabras clave: Ancianos, Calidad de vida, Salud.

\section{INTRODUÇÃO}

A Qualidade de Vida (QV) insere-se em um conceito subjetivo que depende do estado de saúde, as relações familiares, longevidade, disposição, lazer, satisfação no trabalho, salário, prazer, espiritualidade, capacidade funcional, enfraquecimento, deficiência, nível de atividade física, parâmetros antropométricos e uso de medicamentos (PEREIRA DS, et al., 2015).

No Brasil a QV tornou-se ponto de estudos nos últimos anos por conta do crescimento demográfico que desencadeou o aumento expressivo da população idosa no país. O conceito de QV perdura em constante atualização, por conta de que se trata de um conjunto multidisciplinar, que tem em comum dois pontos: a subjetividade e a multidimensionalidade (FERREIRA LK, et al., 2018).

A Organização Mundial da Saúde (OMS) define a QV como a percepção do indivíduo sobre sua posição na vida, no contexto cultural, bem como no sistema de valores em que vive, assim como em relação aos seus objetivos, expectativas, padrões e preocupações. Dessa maneira, a OMS propõe que para se avaliar a QV de forma confiável, é necessário que se aborde aspectos biológicos, psicológicos, sociais, ambientais e espiritual, incluindo um sistema de valores que envolvam metas, expectativas, padrões e preocupações (SARDINHA AHL, et al., 2019).

A QV envolve uma série de fatores que interligados proporcionam ao idoso um favorável bem-estar, como: o relacionamento interpessoal, manutenção de boa saúde, equilíbrio emocional, preservação de bens materiais, lazer, trabalho que propicie satisfação, religião, solidariedade e ambiente favorável. Falar, conceituar ou definir QV é totalmente complexo e engloba fatores físicos, psicológicos, emocionais, ambientais dentre outros (ALMEIDA BL, et al., 2020).

Para Paiva FT, et al. (2019) a QV pode ser interferida ou prejudicada devido a uma série de fatores como: doenças crônicas, problemas sócio-sanitários, não prática de atividade física, quedas, capacidade funcional diminuída, falta de autonomia e independência, sexualidade, preconceito, rejeição familiar e perdas. Todos esses fatores tornam-se responsáveis pelo baixo nível de qualidade de vida, infringindo o bom estado biopsicossocial dos idosos.

Quando se falar de QV na terceira idade, lembraremos de: independência e autonomia, continuidade de papeis sociais e de relações sociais e intergerenciais, a aceitação e o envolvimento social, o conforto e a segurança ambiental, a qualidade de vida nas instituições, a presença de condições econômicas que permitam uma vida digna, a disponibilidade de serviços de saúde, o apoio formal e informal a idosos portadores de doenças e incapacidades, e as políticas sociais dirigidas à assistência e ao cuidado dos idosos (LEMOS BO, et al., 2019).

O aumento da população idosa no Brasil é um reflexo do fenômeno do envelhecimento que é realidade no mundo inteiro. Portanto é de extrema importância compreender a pessoa idosa, buscando conhecê-la de 
forma complexa e de acordo com sua realidade, pois dessa forma os profissionais de saúde e o governo poderão traçar planos de ação para melhorar a qualidade de vida do idoso.

Dessa maneira, é necessário que se estude a população longeva, considerando que futuramente o Brasil será o $6^{\circ}$ país do mundo com maior população de idosos. Assim, o estudo tem por objetivo avaliar a qualidade de vida dos idosos de um município do sertão central cearense.

\section{MÉTODOS}

Trata-se de um estudo transversal de abordagem quantitativa. A pesquisa foi realizada em um município no estado do Ceará, Brasil. O mesmo está localizado na região do sertão central cearense, mais precisamente na microrregião dos sertões de Quixeramobim. Com uma área territorial de 956,122 Km²e uma população de 26.469 habitantes, dos quais 3.372 são idosos, o que corresponde a $12 \%$ da população total (IBGE, 2010).

A coleta dos dados foi realizada nos meses de março e abril de 2012. A amostra correspondeu ao número finito de idosos, $n=358$, selecionados de forma aleatória. Porém, pela acessibilidade do pesquisador, a amostra final deu-se pelo $n=370$.

Quanto aos procedimentos para a coleta dos dados, foram aplicados nos domicílios. Os objetivos da pesquisa foram apresentados e explicados, sendo a aplicação da entrevista realizada com autorização do idoso e/ou familiar responsável através da assinatura do Termo de Consentimento Livre e Esclarecido (TCLE).

Foram incluídos na pesquisa pessoas com idade igual ou superior a 60 anos, de ambos os sexos, com a cognição preservada e assim apta a responderem os questionários. Foram excluídos da pesquisa indivíduos com deficiência auditiva e/ou visual ou que não compreendia a comando verbal simples.

Os dados foram coletados através da aplicação de questionários, evidenciado pela coleta do perfil demográfico, socioeconômico e do perfil clínico. Foram coletadas as informações concernentes aos aspectos: sexo, idade, estado civil, grau de escolaridade, renda mensal, presença de comorbidades e estado de saúde e pelo teste que avaliou a qualidade de vida dos idosos, composto por questionamentos interligados aos domínios de capacidade funcional, aspectos físicos, dor, estado geral de saúde, vitalidade, aspectos sociais, aspectos emocionais e saúde mental.

Os dados foram tabulados no Microsoft Office Excel 2007 e tratados estatisticamente utilizando o programa SPSS-Statistical Package for Social Sciences - versão 20.0, para inferências de frequência absoluta, percentual, média e desvio padrão. Para o escore de Qualidade de Vida utilizou-se o Medical Outcome Study 36-item Short Form (SF-36) como instrumento de coleta, por se tratar de um método que melhor avalia a Qualidade de Vida (QV). É constituído por 36 pontos fragmentados em oito domínios: capacidade funcional, aspectos físicos, dor, estado geral da saúde, vitalidade, aspectos sociais, aspectos emocionais e saúde mental. O escore de cada domínio pode variar de 0 a 100, sendo que os escores mais próximos de 0 simbolizam uma pior percepção de qualidade de vida enquanto aqueles mais próximos de 100, uma melhor percepção de qualidade de vida.

A pesquisa teve aprovação do Comitê de Ética da Faculdade Católica Rainha do Sertão - FCRS sob número de protocolo 20120017.

\section{RESULTADOS}

Total de 370 idosos, destes 258 (69,7\%) são mulheres e $112(30,3 \%)$ homens. A análise da idade expôs uma média de 71,48 anos e $\mathrm{DP}=9,27$, observando-se o perfil dos idosos da quarta idade (idosos com 80 anos ou mais) que compreendem $73(19,7 \%)$. Dos idosos constituintes da pesquisa, $296(80 \%)$ residem na zona urbana e $74(20 \%)$ na zona rural. A raça branca é predominante entre os entrevistados $186(50,27 \%)$, parda 115 (31,08\%) e negra 68 (18,38\%); Estado civil predominou casado 193 (52,16\%) e viúvo 103 (27,88\%); Escolaridade predominou analfabeto $180(48,65 \%)$ e fundamental incompleto 160 (43,24\%); Religião católico 329 (88,92\%) e evangélico 35 (9,46\%); Aposentados 339 (91,62\%) e ocupação agricultor 221 (59,73\%).

O idoso do sexo masculino possui uma QV melhor do que as mulheres, isso tanto para o aspecto físico, quanto para o mental. Os resultados apontam uma diferença significativa entre os sexos, onde os níveis de 
qualidade para homens são de 53,35\% e para mulheres de $49,73 \%$. Entre o gênero masculino e feminino a caracterização da QV dos idosos se deu pela avaliação de aspectos como capacidade funcional, aspecto físico, dor, aspecto geral de saúde, vitalidade, aspecto social, aspecto emocional e a saúde Mental (Tabela 1).

Tabela 1- Categorias da qualidade de vida dos idosos.

\begin{tabular}{cc}
\hline Categorias & Pontuação (0-100) \\
\hline Capacidade Funcional & 69 \\
Aspecto físico & 19 \\
Dor & 59 \\
Aspecto geral de saúde & 51 \\
Vitalidade & 66 \\
Aspecto social & 83 \\
Aspecto emocional & 81 \\
Saúde Mental & 70 \\
Coeficiente Físico Sumarizado (CFS) & 45 \\
Coeficiente Mental Sumarizado (CMS) & 50 \\
\hline
\end{tabular}

Fonte: Ponciano DR, et al., 2020.

O estudo mostra em seus resultados que o idoso sertanejo que possui uma renda baixa, ou seja, de até um salário mínimo são aqueles que apresentam os piores níveis de QV, tanto no aspecto físico quanto no emocional. Os resultados ainda mostram que quanto maior a renda do idoso, maior será sua QV. O número de idosos com uma elevada renda mensal é mínimo, o que significa dizer que a grande maioria dos idosos do município em estudo encontra-se com renda de até no máximo dois salários, o que pressupõe que a QV poderá ser afetada. A religião católica é extremamente presente no contexto do idoso nordestino.

Interessante ressaltar que os resultados do estudo mostram que os idosos que possuem um nível de escolaridade maior, vivem com uma QV melhor do que os idosos analfabetos. Quanto ao grau de escolaridade percebe-se que os idosos que tem um nível educacional elevado, elevado também é sua QV. Os idosos que possuem ensino médio completo e incompleto e superior completo obtiveram os melhores resultados.

Ao que diz respeito à convivência familiar, o presente estudo mostra que os idosos que avaliaram como ótimo a convivência com a família são os que vivem melhor, isso no aspecto mental, pois no aspecto físico o índice de QV foi negativo. Os idosos que obtiveram o pior resultado no aspecto mental foram aqueles que apontavam ter um péssimo convívio familiar, porém estes foram os que apresentaram o melhor nível de QV no aspecto físico.

\section{DISCUSSÃO}

Segundo Manso MEG, et al. (2019), as mulheres vivem melhor do que os homens isso ao que diz respeito a população geriátrica. Os autores trazem que o fato de os homens serem o chefe da casa, carrega uma maior sobrecarga de estresse, possui hábitos de vida não saudáveis e tudo isso contribui para que na velhice eles sofram um impacto negativo em sua saúde.

A saúde da mulher há tempos é ponto de estudos pela área da saúde. Na atenção básica projetos existem para prevenir doenças e agravos e para prover saúde para esse grupo específico, e mesmo assim as mulheres acabam que tendo uma QV diminuída se comparada com os homens. A saúde do homem é recente para os profissionais de saúde, o ministério da saúde lançou a Política Nacional de Atenção Integral à Saúde do Homem para que os homens possam também ser assistidos de acordo com suas particularidades. Nesse sentido, a política representa uma pretensão da sociedade ao reconhecer que os agravos do sexo masculino constituem verdadeiros problemas de saúde pública. Porém fica o questionamento: Porque as mulheres ainda não conseguem uma boa QV mesmo com a atenção básica tendo um de seus focos direcionados para elas? (BRASIL,2016).

Segundo Sardinha AHL, et al. (2019), o baixo nível educacional é um dos fatores para que a QV seja baixa. Outros autores também quando pesquisam sobre QV voltada a população idosa chegam à conclusão em seus resultados que a escolaridade é um quesito que pode determinar uma QV positiva ou negativa. 
Pesquisadores corroboram o fato de que o grau de instrução proporciona os idosos a buscarem mais os serviços de saúde e o conhecimento facilita uma busca pela melhora e recuperação da saúde (MANSO MEG, et al., 2019).

O nível educacional da pessoa idosa torna-se um dos fatores a serem investigados em uma avaliação multidimensional em virtude de ser responsável por facilitar ou dificultar a compreensão das atividades e orientações recebidas pelo idoso. Entende-se que o nível de escolaridade influencia os fatores de risco para quedas, as formas de prevenção destas, e consequentemente as implicações na QV (PAIVA MM, et al., 2020).

A pesquisa realizada pelo IBGE constata que uma das características da população idosa brasileira é a presença do baixo nível de escolaridade. Este fato é registrado em $25 \%$ da população idosa brasileira como analfabetos. Dessa forma, é possível inferir que quanto maior o nível educacional, maior a noção do mundo e da avaliação da própria QV (IBGE, 2013).

A renda do idoso é um dos principais fatores que refletem diretamente na QV. Portanto, para os idosos que possuem um nível econômico baixo, isso pode ter um impacto negativo em sua saúde. Os programas sociais do governo ajudam a amenizar essa realidade, porém recusa extingue o problema, além desse fato diferenciar-se de idoso para idoso (SARDINHA AHL, et al., 2019).

Estudos revelam que o fator econômico tem um grande impacto na QV. Idosos que possuem um nível econômico alto têm fortes chances de terem uma boa QV. Já os idosos que sobrevivem com uma renda baixa são fortes candidatos a terem um nível muito baixo de QV. Isso por conta de que o nível socioeconômico proporciona aos idosos melhores condições de moradia e alimentação, acesso aos serviços de saúde, proporciona ainda atividades de lazer, dentre outras coisas (FERREIRA LK, et al., 2018).

Moreira AAO, et al. (2018), relatam que a aposentadoria traz inúmeros benefícios aos idosos, proporcionando a eles uma melhora no estilo de vida. Porém, para uns idosos o fato de estar aposentado significa não ter mais projetos de vida, além disso, para outros a aposentadoria acaba sendo outra fonte de renda para a família e o idoso acaba sendo menos beneficiado, o que irá resultar em um nível baixo de QV. Então, compete a cada idoso saber aproveitar a aposentadoria como algo a seu benefício.

Os idosos da raça branca são os que apresentam os melhores resultados de QV seguidos pelos idosos negros. A raça amarela apresenta um baixo índice de QV, segundo o estudo em questão. Poucos estudos relacionam a $Q V$ com a raça. Pesquisadores atribuem o idoso negro com uma baixa $Q V$ por geralmente este se enquadrar em outros fatores como: nível de escolaridade, serem agricultores, baixa renda, número elevado de mulheres. Esses fatores que juntos podem ter um grande impacto negativo na QV do idoso (SANTOS VC, et al., 2016).

Nunes MGS, et al. (2017) ressaltam em seu estudo o elevado número de idosos que são adeptos ao catolicismo. Mesmo com o crescimento do protestantismo no Brasil, o idoso ainda mantém sua fé baseada na religião, por conta de questões culturais. De acordo com os autores a população idosa brasileira é bem adepta a religião católica, seus achados mostram essa realidade de forma exacerbada, pois foi expressa por um número de $94 \%$.

Moreira AAO, et al. (2018) afirmam que a convivência familiar é um dos fatores que podem influenciar numa boa QV do idoso. Pois é justamente na velhice que os indivíduos ficam mais carentes e precisam de mais atenção e afeto por parte da família, e muitas vezes não são correspondidos, e assim alguns deles já interpretam como se fossem desprezados pela família. Esse fato pode desencadear um processo de depressão e consequentemente acarretarem uma péssima QV.

Pesquisadores apontam que a atividade física é fundamental para a manutenção de uma boa QV. Idosos ativos tendem a viver melhor do que idosos inativos. A atividade física proporciona ao idoso inúmeros benefício, dentre eles uma sensação de bem-estar que repercute de forma positiva em sua saúde. Em um estudo realizado com idosos praticantes de atividade física, os resultados surpreenderam, onde os índices de QV dos idosos ultrapassaram as expectativas (FERRETI F, et al., 2018).

Janini JP, et al. (2015) fez um estudo comparativo entre idosos ativos e sedentários, e os resultados foram claros. Idosos praticantes de atividade física obtiveram um nível de QV bastante elevado, já aqueles que eram 
sedentários os resultados foram explícitos: péssima QV. O presente estudo também mostra em seus achados que os idosos que praticam atividade física possuem QV melhor do que os sedentários, tanto no aspecto mental quanto no físico. Dessa forma podemos perceber a importância da atividade física para grupos de terceira idade. Infelizmente os idosos do município em estudo em sua grande maioria são idosos sedentários, pois apenas uma pequena minoria é ativa e praticam atividade física.

O lazer é um fator que pode influenciar de forma direta na QV da população idosa. O presente estudo mostra que os idosos que possuem atividades de lazer são aqueles que vivem melhores, onde sua QV é satisfatória em relação aos idosos que não tem lazer. Apenas no aspecto mental os idosos que tem lazer tem uma boa QV, já no aspecto físico o resultado não foi satisfatório, e os idosos que não tem lazer o aspecto mental ficou bem na média e o aspecto físico deixou muito a desejar. De modo geral ambos os idosos (os que têm ou não lazer) estão com QV diminuída, porém os que realmente relatam não terem lazer apresentam números ainda menores (FERREIRA LK, et al., 2018).

Lemos BO, et al. (2019) mostram em seus achados que os idosos que apresentam atividades de lazer possuem uma QV satisfatória, principalmente no aspecto mental, pois proporciona a eles um momento de bem-estar. Por outro lado, situações como abandono, isolamento social, incapacidade de retorno as atividades produtivas que atinjam os idosos acabam sendo fatores que comprometem a QV $e$ consequentemente podem aumentar as chances de desenvolvimento de morbidades psíquicas (SILVA PADSD, et al., 2018; BUSO ALZ, et al., 2020).

A prática de atividade física também se mostra relevante e benéfica para a saúde mental de pessoas idosas. O idoso que pratica atividade física tende a ter menores chances de desenvolver depressão, dentre outras patologias psiquiátricas, em virtude da atividade física servir como ativador de mediadores químicos com efeito calmante e relaxante (MAZO GZ, et al., 2009; GATO JM, et al., 2018).

Ferreti $F$, et al. (2018) ressalta a importância do lazer na vida do idoso, visto que a QV engloba uma série de fatores que irá repercutir em um resultado. Varela DSS (2011) relata que o lazer é algo complexo e subjetivo, e varia de idoso para idoso, porém é fato afirmar que uma vez o idoso identificando alguma atividade como lazer, esta sem dúvidas o trará uma satisfação e bem-estar.

A saúde da pessoa idosa acaba sendo um dos fatores mais importantes para que se tenha uma boa qualidade de vida e bem-estar. No processo de envelhecimento, em que as limitações e incapacidades inerentes ao processo começam a aparecer, ter uma boa saúde é primordial para que a pessoa idosa vivencie um processo de envelhecimento ativo, autônomo e com qualidade (DIAS EN, RIBEIRO JLP, 2018; BRAGA MCP, et al., 2011).

Todos os aspectos expostos são fatores que podem influenciar de forma direta na $Q V$ da população idosa, proporcionando a eles na grande maioria resultados ruins. É verdade afirmar que o idoso da zona rural possui suas particularidades, porém essas características que fazem com que essa população tenha uma QV prejudicada e diminuída, contudo, essa realidade se difere de acordo com as regiões.

\section{CONCLUSÃO}

Conclui-se que na amostra desta pesquisa os homens idosos possuem uma qualidade de vida melhor do que as mulheres idosas, concernente aos aspectos físico e mental. O estudo ressalta os componentes que influenciam na qualidade de vida dos idosos, evidenciando suas conjunções aos aspectos biopsicossocio espirituais que formam o cenário onde esses indivíduos estão inseridos. Assim observam-se algumas limitações, que precisam ser levadas em consideração na interpretação dos resultados sobre domínios da qualidade de vida de idosos. Assim faz-se necessário conhecer esse idoso de forma multifacetada, pois o Nordeste destina-separa uma explosão demográfica que reflete na transformação da pirâmide etária e por consequência tem-se o aumento da população idosa.

\section{AGRADECIMENTOS E FINANCIAMENTO}

Agradecemos aos idosos do município que colaboraram na pesquisa. 


\section{REFERÊNCIAS}

1. ALMEIDA BL, et al. Qualidade de vida de idosos que praticam atividade física, R. pesq. cuid. fundam. online. 2020; 12: $466-470$.

2. BUSO ALZ, et al. Fatores associados à qualidade de vida dos idosos octogenários da zona rural de Uberaba/MG. Cad. saúde colet. Aheadof Print. 2020.

3. BRAGA MCP, et al. Qualidade de vida medida pelo Whoqol-bref: estudo com idosos residentes em Juiz de Fora - MG. Revista de Atenção Primária à saúde. 2011: 14;93-100.

4. BRASIL. Ministério da Saúde. Protocolos da Atenção Básica: Saúde das Mulheres / Ministério da Saúde, Instituto Sírio-Libanês de Ensino e Pesquisa - Brasília: Ministério da Saúde, 2016. 230p.

5. DIAS EM, RIBEIRO JLP. Espiritualidade e qualidade de vida de pessoas idosas: um estudo relacional. Psic., Saúde \& Doenças, 2018; 19 (3):591-604.

6. FERREIRA LK, et al. Avaliação do estilo e qualidade de vida em idosos: uma revisão de literatura, Rev. Bras. Geriatr. Gerontol., Rio de Janeiro, 2018; 21(5): 639-651.

7. FERRETTI F, et al. Qualidade de vida de idosos com e sem dor crônica, Br J Pain. São Paulo, 2018;1(2):111-5.

8. INSTITUTO BRASILEIRO DE GEOGRAFIA E ESTATÍSCA - IBGE. 2010. In: conheça estados e cidades o Brasil.

9. INSTITUTO BRASILEIRO DE GEOGRAFIA E ESTATÍSTICA - IBGE. Estatísticas sobre a população 2013. 2013.

10. JANINI JP, et al. Educação em saúde e promoção da saúde: impacto na qualidade de vida do idoso, Saúde Debate, Rio de Janeiro, abr-jun 2015; v. 39, n. 105, p.480-490.

11. LEMOS BO, et al. O impacto da dor crônica na funcionalidade e qualidade de vida de idosos, BrJP. São Paulo, 2019;2(3): 237-41.

12. MANSO MEG, et al. Análise da qualidade de vida e fatores associados em um grupo de idosos vinculados ao setor suplementar de saúde da cidade de São Paulo, Brasil, Rev. Bras. Geriatr. Gerontol. 2019; 22(4):e190013.

13. MAZO GZ, et al. Do diagnóstico à ação: grupo de estudos da terceira idade: alternativa para a promoção do envelhecimento ativo. RBAFS. 2009;14(1):65-70.

14. MOREIRA AAO, et al. Qualidade de vida e fatores associados em aposentados por invalidez de uma universidade pública brasileira, ciencia y enfermeria, 2018; $24: 4$.

15. NUNES MGS, et al. Idosos longevos: avaliação da qualidade de vida no domínio da espiritualidade, da religiosidade e de crenças pessoais, Saúde Debate, Rio de Janeiro, 2017; 41(115): 1102-1115.

16. PAIVA FTF, et al. A influência da dor na qualidade de vida de idosos portadores de Diabetes Mellitus, Rev enferm UERJ, Rio de Janeiro, 2019; 27: e31517.

17. PAIVA MM, et al. Desigualdades sociais do impacto das quedas de idosos na qualidade de vida relacionada à saúde. Ciênc. Saúde coletiva, 2020:25(5):1887-1896.

18. PEREIRA DS, et al. Qualidade de vida e situação de saúde de idosos: um estudo de base populacional no Sertão Central do Ceará, Rev. Bras. Geriatr. Gerontol., Rio de Janeiro, 2015; 18(4): 893-908.

19. SANTOS VC, et al. Condições socioeconômicas e de saúde associadas à qualidade de vida de idosos quilombolas, Texto Contexto Enferm, 2016; 25(2):e1300015.

20. SARDINHA AHL, et al. Qualidade de vida em idosos quilombolas no nordeste brasileiro, Rev. Bras. Geriatr. Gerontol, 2019; 22(3): e190011.

21. SILVA PADSD, et al. Prevalência de transtornos mentais comuns e fatores associados entre idosos de um município do Brasil. Cien. Saúde Colet. 2018;23(2):639-46.

22. VARELA DSS. Relação do lazer e da qualidade de vida sob a perspectiva do idoso. Monografia (Pós-graduação em saúde do Idoso) Universidade estadual do Ceará. Fortaleza, CE, 2011. 